\title{
Coevolutionary dynamics shape the structure of bacteria-phage infection networks
}

\begin{abstract}
Coevolution-reciprocal evolutionary change among interacting species driven by natural selection - is thought to be an important force in shaping biodiversity. This ongoing process takes place within tangled networks of species interactions. In microbial communities, evolutionary change between hosts and parasites occurs at the same time scale as ecological change. Yet, we still lack experimental evidence of the role of coevolution in driving changes in the structure of such species interaction networks. Filling this gap is important because network structure influences community persistence through indirect effects. Here we quantified experimentally to what extent coevolutionary dynamics lead to contrasting patterns in the architecture of bacteria-phage infection networks. Specifically, we look at the tendency of these networks to be organised in a nested pattern by which the more specialist phages tend to infect only a proper subset of those bacteria infected by the most generalist phages. We found that interactions between coevolving bacteria and phages become less nested over time under fluctuating dynamics, and more nested under arms race dynamics. Moreover, when coevolution results in high average infectivity, phages and bacteria differ more from each other over time under arms race dynamics than under fluctuating dynamics. The trade-off between the fitness benefits of evolving resistance/infectivity traits and the costs of maintaining them might explain these differences in network structure. Our study shows that the interaction pattern between bacteria and phages at the community level depends on the way coevolution unfolds.
\end{abstract}

\section{antagonistic interactions | ecological networks | community structure host range | specialization | resistance}

This is the author manuscript accepted for publication and has undergone full peer review but has not been through the copyediting, typesetting, pagination and proofreading process, which may lead to differences between this version and the Version of Record. Please cite this article as doi: $10.1111 /$ evo.13731

This article is protected by copyright. All rights reserved. 


\section{Introduction.}

2 The ecological importance of coevolution (i.e., reciprocal evolutionary change be-

3

4

5

6

7

8

9 tween interacting species driven by natural selection; Thompson 2005) relies on the

ways coevolutionary dynamics shape the structure of biodiversity. For example, previous theoretical studies have suggested that coevolution within mutualistic communities can drive changes in trait distributions and hence, might shape the patterns of interdependencies among species (Nuismer et al. 2013; Guimarães et al. 2017). Yet, none of the current ecological models of antagonistic interactions can be used directly to evaluate the effects of coevolutionary dynamics on the structure of phenotypic diversification (see however Hochberg and van Baalen 1998). Building a strong theory of the ecological consequences of coevolutionary dynamics requires the design of experimental systems that provide insights and guide the development of theoretical approaches.

The life cycles and antagonistic interactions of bacteria and lytic phages make microbial communities a powerful model system to explore the role of coevolution in shaping ecological patterns because changes in gene frequencies take place at the same time scale as changes in population abundances (Betts et al 2016; Bohannan and Lenski 2000; Weitz et al. 2013). If changes in gene frequencies translate into phenotypic trait changes that affect demographic rates (such as reproduction or survival), then, ultimately, the genetic change will affect population dynamics. Phages infect their bacterial hosts by attaching to cell surface receptors and one way for bacteria to evolve resistance is by modifying or eliminating the attachment sites. The mutations responsible for these modifications may simultaneously reduce the bacteria's competitiveness because the receptor molecules are often in- 
volved in resource acquisition (Lenski 1988). Phages, in turn, can evolve reciprocal adaptations to circumvent host resistance (Meyer et al. 2012).

Cross-infection experiments across time (i.e., time-shift assays) were initially applied by Buckling and Rainey (2002) to distinguish arms race dynamics (i.e., hosts become resistant to a wider range of parasite genotypes and parasites evolve the ability to infect a wider range of host genotypes across time) from fluctuating dynamics (i.e., different, rather than greater, resistance and infectivity profiles are alternatively favoured through time). Under fluctuating dynamics (also called Red Queen dynamics), natural selection favors host genotypes that are rare if they can escape attack by parasites that are locally adapted to the most common host genotype (Ashby and Boots 2017, Best et al. 2017). At the same time, selection will continue favoring parasites capable of attacking the most common hosts. In contrast, arms race dynamics are driven by directional selection toward an everincreasing investment in host defense and parasite counterdefense (Buckling and Rainey 2002; Brockhurst et al. 2003; Scanlan et al. 2011).

Early theoretical (Hochberg and van Baalen 1998) and experimental (LopezPascua et al. 2009) studies have suggested that the level of resources available for hosts shapes the outcome of coevolution. It has been suggested that the mechanism responsible for the influence of resources on coevolutionary dynamics is the cost of mutating receptors, with a lower cost when nutrients are more abundant (LopezPascua and Buckling, 2008). What remains to be investigated is to what extent differences in coevolutionary dynamics lead to contrasting patterns in the structure of bacteria-phage infection networks.

A bacteria-phage infection network depicts who infects whom as links connect 
susceptible bacteria to the phages that infect them (i.e., nodes of the network). The structure of such a network is characterized by the pattern of links established among all coevolving phages and bacteria that are present in the community at a given time. Quantifying network structure in microbial and viral communities is highly relevant because community assembly models rarely account for the influence of evolutionary change on ecological dynamics. For example, phages may infect a single, unique bacterial phenotype or may diversify and result in nested networks in which the most specialist phages infect those hosts that are most susceptible to infection rather than infecting those hosts that are most resistant to infection (see insets on Fig. 3). This nested pattern was first described in the context of plant-animal mutualistic networks (Bascompte et al. 2003), and posteriorly applied to bacteria-phage infection networks (Flores et al. 2011). The relevance of looking at this network pattern hinges on the fact that it may affect both the number of coexisting species supported by these networks (Bastolla et al. 2009) as well as their robustness in the face of perturbation (Rohr et al. 2014).

In a first attempt to provide empirical evidence on how the level of resources available for hosts influences network structure by shifting coevolutionary dynamics, Poisot et al. (2011) found that nestedness was greater at low than at high resources. However, this study lacked competition among both bacteria and phages because it was performed on a collection of pairwise bacteria-phage coevolving populations. Only recently this question has been addressed in experimental bacteriaphage infection networks (Gurney et al. 2017). The authors used a previous study (Betts et al. 2014) to test whether the networks resulting from coevolving populations that exhibited arms race dynamics were more nested than networks resulting 
from fluctuating dynamics. No differences were found in terms of structure between the networks resulting from the two modes of coevolutionary dynamics. However, a limitation of their approach is that they used phages from different families coevolving with the same bacteria species. This precludes exploring how coevolution shapes network structure within the same bacteria-phage system.

Here we go further along this path in two novel directions. First, we shift the focus from genotypic to phenotypic coevolution. Isolates sampled from the coevolving population at different times might correspond to the same genotypes (likely the most abundant ones). Since we are interested in phenotypic evolution, we circumvented this uncertainty by focusing on the unique phenotypes for both bacteria and phages. This will allow us to minimize the effects of differences in genotype abundance (i.e., the ecology of the system) and focus on the evolutionary dimension. Characterizing coevolutionary dynamics at the phenotype level is important because abundance may explain asymmetries in bacteria-phage interactions (i.e., phages of the abundant phenotypes will have frequent encounter with bacteria of many rare phenotypes). Second, we quantify changes in the structure of the interaction network at two levels. We begin by looking at the contemporary interaction networks at each time step. This will allow us to explore to what extent the coevolutionary mode shapes network structure. We then proceed by considering, for each replicate, the global network of interactions accumulated across the entire experimental setting, which will allow us to see to what degree the phenotypes of the contemporary networks are more or less similar across time. Hereafter, we will refer to the former scale as the contemporary network and to the latter scale as the global (contemporary plus non-contemporary) network. As 
a model system, we look at the structure of the network resulting from the phenotypic diversification in a pairwise coevolutionary framework, where a single phage species (SBW25ф2) infects one host bacterium species (Pseudomonas fluorescens SBW25) in high and low nutrient environments (Lopez-Pascua et al. 2014).

\section{Methods.}

\subsection{Coevolutionary experiments.}

We used data from the coevolutionary experiment carried out by Lopez-Pascua et al. (2014) using P. fluorescens SBW25 and phage SBW25 $\phi 2$. They cultivated 12 coevolving populations of bacteria and their phages during 24 days in 2 different nutrient environments ( 6 with high and 6 with low resource availability). The high and low nutrient media contained the same nutrients (proteose peptone and glycerol), but with 10-fold difference in concentration. The same receptors should therefore be expressed in the bacteria. While we do not know the precise binding site of the phage, characterization of resistant bacteria suggests phages bind to lipopolysaccharides on the bacteria outer membrane (Scanlan et al., 2015). Then, they isolated 20 bacteria and 20 phages every 4 days (i.e., 6 times for the entire coevolutionary process; Fig. 1a). Using those isolates, the infectivity or resistance of every pairwise bacterium-phage combination within each of the 12 populations was tested (i.e., $(20 \times 6) \times(20 \times 6)=14400$ infectivity and resistance assays per population; Fig. 1b). Further details on the evolution experiment, the procedure to isolate coevolved bacteria and phages, and how infectivity and resistance assays were performed can be found in Lopez-Pascua et al. (2014). 


\subsection{Phenotype-based bacteria-phage infection networks.}

We first assigned, for each replicate and resource level, a single phenotype to each of the 20 phages and 20 bacteria isolated in the lab at each point in time by identifying their unique infectivity (phages) or resistance (bacteria) profiles. These profiles result from testing the outcome of the $(20 \times 6) \times(20 \times 6)=14400$ pairwise cross-infections for each replicate. That is, we assigned the same phenotype to two phages (bacteria) if they showed the same infectivity (resistance) profile against all bacteria (phages) isolated during the entire coevolutionary process (Fig. 1c). This mapping of genotypes onto phenotypes resulted in infectivity matrices between one-third and a half the size of the $120 \times 120$ pairwise cross-infections (mean and standard deviation for the number of unique infectivity (resistance) profiles of phages (bacteria) was $53.8 \pm 35.8(39.8 \pm 24.7)$ at low nutrients, and $63.2 \pm 24.4$ $(36 \pm 12.8)$ at high nutrients).

Second, for each replicate and resource level, we redrew the $20 \times 20$ infectivity matrices of bacteria and phages isolated at time $t$ by keeping only those bacteria and phages with unique phenotypes (contemporary networks; Fig. 1d). Note that, if a bacterium or phage with the same phenotype was sampled at more than one point in time, the same phenotype will be found in more than one contemporary network. In addition, some bacteria and/or phages from a contemporary network might not have any interactions just because of the sampling process. This does not mean those bacteria had evolved resistance to all phages, but only to the phages isolated at time $t$. Likewise, those phages might not be able to infect any of the bacteria isolated at time $t$, but they would be able to infect other bacteria in the population - otherwise they would not have been sampled. We included those 
phenotypes in the analyses of the contemporary networks because they affect the average infectivity of the coevolving population.

Third, we redrew the infectivity matrices consisting of all pairwise cross-infections for each replicate and resource level (i.e., global networks; Fig. 1d) by considering those bacteria and phages of their corresponding contemporary networks. As noted above, a global network might contain more than one bacterium and/or phage with the same phenotype if they were sampled at more than one point in time. We included them in our analyses to infer coevolutionary dynamics (see below), but kept only the isolate that was sampled first as the unique phenotype in the other analyses. This ensured that we matched the unique phenotypic characterization to the temporal sequence of the coevolutionary process.

Finally, to infer coevolutionary dynamics at the phenotype level (Fig. 2), the pairwise interactions (i.e., phage phenotype $i$ infecting bacterium phenotype $j$ ) from each global network were classified into three groups: 1) interactions among contemporary bacteria and their coevolving phages (i.e., phage phenotype $i$ sampled at time $t$ was able to infect bacterium phenotype $j$ sampled at time $t$ ); 2) interactions among phages sampled from future points in time and bacteria sampled from past points in time (e.g., phage phenotype $i$ sampled at time $t+1$ was able to infect bacterium phenotype $j$ sampled at time $t$ ); and 3 ) interactions among phages sampled from past points in time and bacteria sampled from future points in time (e.g., phage phenotype $i$ sampled at time $t-1$ was able to infect bacterium phenotype $j$ sampled at time $t$ ). Since the same phenotype can be sampled at more than one point in time, we kept the first occurrence of the pairwise interaction to ensure that each interaction was represented only once in the data set. 


\subsection{Statistical analysis.}

\subsubsection{Phenotypic diversification and betadiversity.}

Phenotypic diversification was computed by counting the number of novel infectivity and resistance profiles (phage and bacteria phenotypes, respectively) identified at each point in time, replicate, and resource level. We used a linear mixed model to test the effect of resources on phenotypic diversification. We specified resources, time, type of organism (either phage or bacterium), and their interaction as fixed effects, and included replicate as a random effect. We used the type I analysis of variance to quantify the effects of the predictors (Kenward-Roger approximation).

Beta-diversity (i.e., changes in phenotypic composition over time) was quantified following a method that allows us to decompose the contribution of two additive components - phenotype replacement over time (i.e., turnover) and phenotype loss or gain - to beta-diversity patterns (Baselga 2010). We used a linear model to analyze the effect of resources, type of organism, and their interaction on the total beta-diversity and on the fraction of the total beta-diversity explained by phenotypic turnover.

\subsubsection{Phage infectivity to evolving and coevolving bacteria.}

Interactions between unique phenotypes of bacteria and phages were identified by pairwise cross-infection assays (i.e., phage isolate having phenotype $i$ infected bacterium isolate having phenotype $j$ in the cross-infection assay). Phage infectivity was also computed separately for the three types of interactions: interactions among coevolving bacteria and phages, interactions among phages sampled from future points in time and bacteria sampled from past points in time, and phages sampled from past points in time and bacteria sampled from future points in time. 
The role of resources in explaining the probability for a phage to infect a coevolving bacterium compared to that of infecting a bacterium either from the past or from the future was analyzed using a generalized linear mixed model. We modeled the probability of infection with a binomial distribution (link function=logit). We specified the statistical interaction between the type of interaction and the resource level as fixed effects, and we included replicate as a random effect. We used the type I analysis of variance to quantify the effects of the predictors (Kenward-Roger approximation). Here, by type of interaction we refer to the temporal dimension, i.e., contemporary bacteria and phage, bacteria from the future and phage from the past, and viceversa.

\subsubsection{Nestedness.}

We computed nestedness in the pattern of interactions among bacteria and phages for the global and contemporary networks. We used a slightly modified version of the metric introduced by Bastolla et al. (2009) that measures the average overlap betweeen the infectivity (susceptibility) profiles of phages (bacteria). It is equivalent to the widely-used NODF metric (Almeida-Neto et al., 2008), but without penalizing the contribution to nestedness of phages (bacteria) able to infect (susceptible to) the same number of bacteria (phages). Specifically, nestedness was computed as:

$$
N=\frac{\sum_{i=1, i<j}^{b} \frac{m_{i j}}{\min \left(m_{i}, m_{j}\right)}+\sum_{i=1, i<j}^{p} \frac{n_{i j}}{\min \left(n_{i}, n_{j}\right)}}{\frac{b \times(b-1)}{2}+\frac{p \times(p-1)}{2}},
$$

where $b$ is the number of bacteria, $p$ is the number of phages, $m_{i}$ is the number of 
phages infecting bacterium $i, n_{i}$ is the number of bacteria that phage $i$ infects, $m_{i j}$ is the number of common phages infecting bacteria $i$ and $j$, and $n_{i j}$ is the number of common bacteria that phages $i$ and $j$ infect. Nestedness defined above is zero if $m_{i j}=0$ and $n_{i j}=0$ (i.e., no common interactions among bacteria nor among phages), and one (i.e., perfect nestedness if bacteria share all the phages they are susceptible to, and phages share all the hosts they infect) if $m_{i j}=\min \left(m_{i}, m_{j}\right)$ and $n_{i j}=\min \left(n_{i}, n_{j}\right)$.

The absolute values of nestedness resulting from this equation (as well as for the NODF metric) depend on network size (i.e., the number of phages multiplied by the number of bacteria) and connectance (i.e., the number of realized interactions over the total number of bacteria-phage pairs). That is, the smaller the number of phenotypes and the larger the number of interactions, the higher the chances for phage (bacteria) infectivity (resistance) profiles to overlap (Almeida-Neto et al. 2008). In contrast to having a single realization resulting from a given level of resources, here we had enough data (i.e., 6 replicates) to explore the effect of the resource level in determining changes in nestedness over time after controlling for network size and connectance. Since $43 \%$ of the contemporary networks were perfectly nested (i.e., $N=1$ ), we first tested the role of network size in explaining the prevalence of perfect nestedness by using a generalized linear mixed model (binomial distribution; link function=logit). We specified network size and the interaction between time and resources as fixed effects, and included replicate as a random effect. Next, we explored changes in connectance over time for each resource level by using a generalized linear mixed model (binomial distribution; link function=logit). We specified resources and the interaction between time and 
resources as fixed effects, and included replicate as a random effect. After that, we focused on contemporary networks that were large enough to allow nestedness to vary (i.e., $N<1$ ). We then used a linear mixed model to analyze the effect of the resource level in determining changes in nestedness (logit-transformed) over time. We specified connectance, network size, and the interaction between time and resources as fixed effects, and included replicate as a random effect. Finally, we used a linear model to analyze the effect of connectance, resources, and their interaction, on the nestedness of the global network. All statistical analysis were conducted in $\mathrm{R}$ version 3.5.0 ( $\mathrm{R}$ Core Team, 2018).

\section{Results.}

\subsection{Phenotypic diversification and beta-diversity.}

Phages diversified more than bacteria $\left(F_{1,10}=18.93, p=0.001\right.$; see Table S1). The number of novel phenotypes (i.e., unique infectivity and resistance profiles) decreased over time $\left(F_{1,116}=31.42, p<0.001\right)$; however, the magnitude of the decay depended on whether the organism was a phage or a bacterium $\left(F_{1,116}=\right.$ 18.01, $p<0.001)$. Specifically, the number of novel phage phenotypes decreased over time slower than bacteria, and much slower under high than low resources $\left(F_{1,116}=12.70, p<0.001\right)$.

Beta-diversity (i.e., changes in phenotypic composition over time) was higher for phages than for bacteria $\left(F_{1,20}=9.08, p=0.007\right.$; see Table S2). We found no effect of the resource level on beta-diversity $\left(F_{1,20}=1.31, p=0.266\right)$. Interestingly, the turnover component of beta-diversity (measured as the fraction of the total beta-diversity explained by phenotypic turnover) was higher for bacteria than for 
phages under low resources $\left(F_{1,20}=7.00, p=0.016\right)$.

\subsection{Phage infectivity to evolving and coevolving bacteria.}

In addition to previous analysis focused on characterizing coevolutionary dynamics at the genotype level, we identify here the two modes of coevolutionary dynamics at the phenotype level (i.e, regardless of the abundance of their genotypes). The probability of a phage infecting a bacterium depended on the interaction between resources and the type of interaction (i.e., contemporary, bacteria from future and phage from past, or bacteria from past and phage from future; $\chi_{d f=2}^{2}=10.15$, $p=0.006)$. The magnitude and direction of this effect depended on whether bacteria and phages coevolved or bacteria (phages) were facing phages (bacteria) either from the past or the future. Under low resources, bacteria were more resistant to their contemporary than past and future phages, which is consistent with fluctuating dynamics when bacteria adapt more rapidly than do phages (Fig. 2). In contrast, at high resources bacteria were more resistant to past phages and became less resistant to contemporary and future phages, which is a distinctive feature of arms race dynamics (Fig. 2). Indeed, bacteria sampled at the end of the experiment (i.e., $\mathrm{t}=6$ ) evolved resistance to all sampled contemporary phages in $83 \%$ of the replicates under high resources, but only in $33 \%$ under low resources.

\section{$3.3 \quad$ Nestedness.}

We found that the probability for a contemporary network to be perfectly nested depended on network size $\left(\chi_{1}^{2}=22.93, p<0.001\right.$; see Table S4). Small networks ( size $<=50$ ) were all perfectly nested, regardless of the mode of coevolution.

Since neither coevolutionary dynamics, nor time explained network connectance 
$\left(\chi_{d f=1}^{2}=1.31, p=0.253\right.$ and $\chi_{d f=1}^{2}=0.02, p=0.879$, respectively; see Table S5), we did not include a three-way interaction term in the model. The change in nestedness over time observed when considering the non-perfectly nested networks depended on coevolutionary dynamics after controlling for network size and connectance $\left(F_{1,9}=21.42, p=0.001 ;\right.$ see Table S6). That is, nestedness decreased over time under fluctuating dynamics and increased over time under arms race dynamics (Fig. 3).

Moving now to patterns in the global network (i.e., both contemporary and noncontemporary phages and bacteria), the nested pattern of bacteria-phage infections depended on the interaction between coevolutionary dynamics and the connectance of the global networks $\left(F_{1,8}=10.89, p=0.011\right.$; see Table S7). Specifically, networks with higher connectances were more nested under fluctuating dynamics than under arms race dynamics (Fig. 4).

\section{Discussion.}

We have shown how coevolutionary dynamics influences the architecture of bacteriaphage infection networks. First, we found that phages diversify more than bacteria and that the turnover is higher for bacteria than for phages under fluctuating dynamics. Second, the two contrasting modes of coevolutionary dynamics (i.e., fluctuating dynamics and arms race dynamics) driven by the level of resources were also found at the phenotype level. Third, the pattern of interactions among bacteria and phages depends on coevolutionary dynamics at two different scales.

At a local scale, the nested pattern of interactions between coevolving bacteria and phages decreases over time (i.e., niche partitioning is promoted) under fluctuating 
dynamics, and increases over time under arms race dynamics (i.e., niche overlap is promoted; Fig. 3). At a global scale, the higher the network connectance, the higher the nestedness under fluctuating dynamics and the lower the nestedness under arms race dynamics (Fig. 4). Let us discuss those main findings one by one.

\subsection{Phenotypic diversification and beta-diversity}

The decrease in phenotypic diversification over time - regardless of the mode of coevolution - might be explained by coevolution proceeding faster earlier (Bohannan and Lenski 1997; Morgan et al. 2010) and resistance mutations with lower cost appearing at later stages (Bohannan and Lenski 2000). Bohannan and Lenski (1997) showed that, in coevolving populations of E. coli and phage T4, multiple resistant types appeared quickly in bacterial populations at both high and low resources. Under these circumstances, the community would initially increase its diversity, as resistant mutants appear and phages evolve counterdefenses. However, this first burst of adaptive radiation would be followed by a period of decelerating coevolution, as resistance mutations with lower cost appear, reducing the size of the phage population and thus its rate of evolution.

Population abundances could explain why phages diversified over time more than bacteria under arms race dynamics (i.e., at high resources). Increasing concentrations of resources leads to an increase in the abundance of the phage and its host (e.g., Bohannan and Lenski 1997; Forde et al. 2008). Furthermore, the cost of mutating the bacterial receptor is lower when nutrients are more abundant (LopezPascua and Buckling, 2008). Since large populations produce more mutants and the cost of resistance is lower, the selective pressure on phages is stronger under arms race dynamics and hence, it is expected a higher diversification over time. 
Phenotypic composition changed very fast over time, suggesting that coevolution occurs with fast rates relative to the generation time (Forde et al. 2004). Moreover, phenotypic turnover in bacteria was greater under fluctuating dynamics than under arms race dynamics, most likely as a consequence of the frequencydependent selection that might take place under fluctuating dynamics - where selection continually favors rare phenotypes and disfavors common phenotypes.

\subsection{Coevolutionary dynamics}

By measuring the change in the infectivity of phage populations to a bacterial population through time, we found a strong signature at the phenotype level in how resources drive coevolutionary dynamics (Fig. 2). Specifically, we found an everincreasing reciprocal investment in defense and counterdefense at high resources (arms race dynamics), and selection favoring alternative phenotypes in bacteria and phages over time at low resources (fluctuating dynamics). Note that characterizing coevolutionary dynamics at the genotype level (i.e., when the frequency of genotypes is considered) did show fluctuating dynamics, but in a different way (see Lopez-Pascua et al. 2014). That is, instead of promoting different phenotypes of bacteria and phages over time, selection favored host range fluctuations (i.e., the most abundant phages shifted between generalists and specialists over time).

\subsection{Network structure}

The way the level of resources modulates the ecological consequences of the cost of resistance and infectivity (Koskella et al. 2012) might explain the decrease in the nested structure of contemporary networks over time under fluctuating dynamics (Fig. 3). Under low resources, bacterial densities are expected to be low, and 
therefore, the likelihood for a phage to encounter a susceptible bacterium would be low. Since evolving infectivity traits likely comes at the price of a slight decrease in the competitive ability for limiting resources (see Bohannan and Lenski 2000), evolving the ability to infect many hosts (i.e., expanded host-range) might come at a higher cost than evolving a single trait to infect only a few (Woolhouse et al., 2001; Leggett et al. 2013). Therefore, natural selection would favor specialization in phages (i.e., niche partioning). This would explain why nestedness decreased over time under fluctuating dynamics. When resources are abundant, the rate of encounters among bacteria and phages will be much higher, and the fitness benefits of establishing a successful infection would overcome the costs of maintaining infectivity traits. This would explain why nestedness increased over time under arms race dynamics.

This result contrasts with the findings by Poisot et al. (2011), who reported high nestedness at low resources (i.e., under fluctuating dynamics). Two points can potentially explain this divergence. First, here we are using unique infectivity profiles, while in Poisot et al. (2011), as in the rest of previous studies, researchers used isolates that may contain the very same genotype. Second, in Poisot et al. (2011) there was competition neither among bacteria, nor among phages, which makes the comparison more difficult.

In addition, these contrasting patterns in nestedness over time are consistent with previous explanations based on the genetic architecture underlying the mechanism of infection (Flores et al. 2011; Beckett and Williams 2013; Weitz et al. 2013; Koskella and Brockhurst 2014). When interactions are driven by a genefor-gene mechanism of infection, mutations in bacteria would confer resistance to 
recently evolved phages while maintaining resistance to past phages. Likewise, phages would evolve infectivity traits without losing the ability to infect ancestral bacteria. Therefore, the set of bacteria that a phage can infect are nested over time. That is, the host-range of the phages are subsets of each other (i.e., niche overlap). This process would lead to nested interaction networks. In contrast, when interactions are driven by a matching-alleles mechanism of infection, bacteria would evolve resistance to a single phage phenotype and would lose any evolved resistance to other phages, whereas mutations in phages would confer infectivity against single bacterial phenotypes at the cost of an entire loss of infectivity against ancestral phenotypes. This process would lead to less nested, or compartmentalized networks (i.e., niche-partioning), where the host-range of the phages are distinct from each other. Interestingly, it is worth noting that experimental studies (Forde et al. 2008) and mathematical models (Hochberg and van Baalen 1998) have suggested that the way the level of resources (and hence, coevolutionary dynamics) affects the cost of resistance depends also on the genetic architecture of the mechanism of infection.

At the level of the global network, the degree of nestedness decreased with connectance under arms race dynamics but increased with connectance under fluctuating dynamics (Fig. 4). Our interpretation is that when coevolution resulted in high average infectivity (i.e., high connectance), bacteria evolved resistance earlier under arms race dynamics than under fluctuating dynamics-because the fitness benefits of resistance would overcome the costs of evolving resistance traits. Therefore, at high resources phages evolved and diversified quickly from the beginning, which allowed them to differentiate from each other over time (i.e., low nested- 
ness). In contrast, at low resources bacteria evolved resistance later on and phages did not have much time to diverge from each other (i.e., high nestedness).

It might be argued that the way we inferred phenotypes from isolates in the lab is misleading. Note, however, that in a previous study, Hall et al. (2011) sequenced the tail fibre gene of the phage and reported that, on average, $40 \%$ of the phage isolates were distinct genotypes. In our study, on average $48 \%$ of the phage isolates were identified as unique infectivity profiles. This result suggests that each distinct infectivity profile (i.e., phenotype) might in fact correspond to a distinct genotype.

Finally, the results here presented have one limitation that is worth stressing. As with all the previous papers on bacteria-phage coevolution, our work is based on isolation-based approaches. Essentially, this means that the interactions within a network are inferred from pairwise cross-infection patterns. As in other fields of ecology and evolutionary biology, our perception is very much constrained by such a pairwise approach. As a consequence, we know very little about what component of species coexistence or coevolutionary dynamics is due to indirect or higher-order effects (Bairey et al. 2016; Levine et al. 2017, Guimarães et al. 2017). Future work should reduce this gap. Only then, we will be well positioned to fully understand the community context of coevolution. 


\section{Supporting Information.}

\section{S1. Supplementary tables.}

S2. Data set.

- database.csv

- phenotypic_diversification.csv

- beta-diversity.csv

- infectivity.csv

- nestedness_global.csv

- nestedness_contemporary.csv

- README.txt

S3. $R$ code.

- R_phenotypic_diversification.r

- $\mathrm{R}$ _betadiversity.r

- R_infectivity.r

- R_connectance.r

- R_nestedness_global.r

- R_nestedness_contemporary.r 


\section{References.}

Almeida-Neto, M., Guimarães, P., Guimarães, P. R. Jr., Loyola, R. D., and W. Ulrich. 2008. A consistent metric for nestedness analysis in ecological systems: reconciling concept and measurement. Oikos 117:1227-1239.

Ashby, B., and M. Boots. 2017. Multi-mode fluctuating selection in host-parasite coevolution. Ecology Letters 20:357-365.

Barey, E., Kelsic, E. D., and R. Kishony. 2016. High-order species interactions shape ecosystem diversity. Nature Communications 7:12285.

Bascompte, J., Jordano, P., Melián, C. J. and J. M. Olesen. 2003. The nested assembly of plant-animal mutualistic networks. Proceedings of the National Academy of Sciences of the United States of America 100:9383-9387.

Bastolla, U., Fortuna, M. A., Pascual-García, A., Ferrera, A., Bartolo, L., and J. Bascompte. 2009. The architecture of mutualistic networks minimizes competition and increases biodiversity. Nature 458:1018-1020.

Baselga, A. 2010. Partitioning the turnover and nestedness components of beta diversity. Global Ecology and Biogeography 19:134-143.

Beckett, S. J. and H. T. P. Williams. 2013. Coevolutionary diversification creates nested-modular structure in phage-bacteria interaction networks. Interface Focus 
3:20130033.

Best, A., Ashby, B., White, A., Bowers, R., Buckling, A., Koskella, B., and M. Boots. 2017. Host-parasite fluctuating selection in the absence of specificity. Proceedings of the Royal Society of London. B. 284:20171615.

Betts, A., Kaltz, O., and M. E. Hochberg. 2014. Contrasted coevolutionary dynamics between a bacterial pathogen and its bacteriophages. Proceedings of the National Academy of Sciences of the United States of America 111:11109-11114.

Betts, A., Gifford, D. R., MacLean, R. C., and K. C. King. 2016. Parasite diversity drives rapid host dynamics and evolution of resistance in a bacteria-phage system. Evolution 70:969-978.

Bohannan, B. J. M., and R. E. Lenski. 1997. Effect of resource enrichment on a chemostat community of bacteria and bacteriophage. Ecology 78:2303-2315.

Bohannan, B. J. M., and R. E. Lenski. 2000. Linking genetic change to community evolution: insights from studies of bacteria and bacteriophage. Ecology Letters 3:362-377.

Brockhurst, M. A., Morgan, A. D., Rainey, P. B., and Buckling, A. 2003. Population mixing accelerates coevolution. Ecology Lettes 11:975-979. 
Buckling, A., and P. B. Rainey. 2002. Antagonistic coevolution between a bacterium and a bacteriophage. Proceedings of the Royal Society of London, B. 269:931-936.

Flores, C. O., Meyer, J. R., Valverde, S., Farr, L., and J. S. Weitz. 2011. Statistical structure of host-phage interactions. Proceedings of the National Academy of Sciences of the United States of America 108:E288-E297.

Forde, S. E., Thompson, J. N., and B. J. M. Bohannan. 2004. Adaptation varies through space and time in a coevolving host-parasitoid interaction. Nature 431:841-844.

Forde, S. E., Thompson, J. N., Holt, R. D., and B. J. M. Bohannan. 2008. Coevolution drives temporal changes in fitness and diversity across environments in a bacteria-bacteriophage interaction. Evolution 62:1830-1839.

Guimarães, P. R. Jr., Pires, M. M., Jordano, P., Bascompte, J., and J. N. Thompson. 2017. Indirect effects drive coevolution in mutualistic networks. Nature $550: 511-514$

Gurney, J., Aldakak, L., Betts, A., Gougat-Barbera, C., Poisot, T., Kaltz, O., and M. E. Hochberg. 2017. Network structure and local adaptation in co-evolving bacteria-phage interactions. Molecular Ecology 26:1764-1777. 
Hall, A. R., Scanlan, P. D., Morgan, A. D., and A. Buckling. 2011. Host-parasite coevolutionary arms race give way to fluctuating selection. Ecology Letters 14:635642.

Hochberg, M. E., and M. van Baalen. 1998. Antagonistic coevolution over productivity gradients. American Naturalist 152:620-634.

Koskella, B., Lin, D. M., Buckling, A., and J. N. Thompson. 2012. The costs of evolving resistance in heterogeneous parasite environments. Proceedings of the Royal Society of London, B. 279:1896-1903.

Koskella, B. and M. A. Brockhurst. 2014. Bacteria-phage coevolution as a driver of ecological and evolutionary processes in microbial communities. FEMS Microbiology Reviews 38:916-931.

Leggett, H. C., Buckling, A., Long, G. H., and M. Boots. 2013. Generalism and the evolution of parasite virulence. Trends in Ecology and Evolution 28:592-596.

Lenski, R. E. 1988. Experimental studies of pleiotropy and epistasis in Escherichia coli. I. Variation in competitive fitness among mutants resistant to virus T4. Evolution 42:425-432.

Levine, J. M., Bascompte, J., Adler, P., and S. Allesina. 2017. Beyond pairwise mechanisms of species coexistence in complex communitites. Nature 546:56-64. 
Lopez-Pascua L., and A. Buckling. 2008. Increasing productivity accelerates hostparasite coevolution. Journal of Evolutionary Biology 21:853-860.

Lopez-Pascua L., Brockhurst M. A., and A. Buckling. 2009. Antagonistic coevolution across productivity gradients: an experimental test of the effects of dispersal. Journal of Evolutionary Biology 23:207.211.

Lopez-Pascua, L., Hall, A. R., Best, A., Morgan, A. D., Boots, M., and A. Buckling. 2014. Higher resources decrease fluctuating selection during host-parasite coevolution. Ecology Letters 17:1380-1388.

Meyer, J. R., Dobias, D. T., Weitz, J. S., Barrick, J. E., Quick, R. T., and R. E. Lenski. 2012. Repeatability and contingency in the evolution of a key innovation in phage lambda. Science 335:428-432.

Morgan, A. D., Bonsall, M. B., and A. Buckling. 2010. Impact of bacterial mutation rate on coevolutionary dynamics between bacteria and phages. Evolution 64:2980-2987.

Nuismer, S. L., Jordano, P., and J. Bascompte. 2013. Coevolution and the architecture of mutualistic networks. Evolution 67:338-354.

Poisot, T., Lepennetier, G., Martinez, E., Ramsayer, J., and M. E. Hochberg. 2011. 
Resource availability affects the structure of a natural bacteria-bacteriophage community. Biology Letters 7:201-204.

R Core Team. 2018. R: A Language and Environment for Statistical Computing (R Foundation for Statistical Computing, Vienna).

Rainey, P. B., and M. J. Bailey. 1996. Physical and genetic map of the Pseudomonas fluorescens SBW25 chromosome. Molecular Microbiology 19:521-533.

Scanlan, P. D., Hall, A. R., Lopez-Pascua, L., and A. Buckling. 2011. Genetic basis of infectivity evolution in a bacteriophage. Molecular Ecology 20:981-989.

Scanlan, P. D., Hall, A. R., Blackshields, G., Friman, V. P., Davis, M. R. Jr., Goldberg, J. B., and A. Buckling. 2015. Coevolution with bacteriophages drives genome-wide host evolution and constrains the acquisition of abiotic-beneficial mutations. Molecular Biology and Evolution 32:1425-1435.

Thompson, J. N. 2005. The geographic mosaic of coevolution. University of Chicago Press. Chicago, IL (USA).

Weitz, J. S., Poisot, T., Meyer, J. R., Flores, C. O., Valverde, S., Sullivan, M. B., and M. E. Hochberg. 2013. Phage-bacteria infection networks. Trends in Microbiology 21:82-91. 
Woolhouse, M. E. J., Taylor, L. H., and D. T. Haydon. 2001. Population biology of multihost pathogens. Science 292:1109-1112. 


\section{$7 \quad$ Figures}

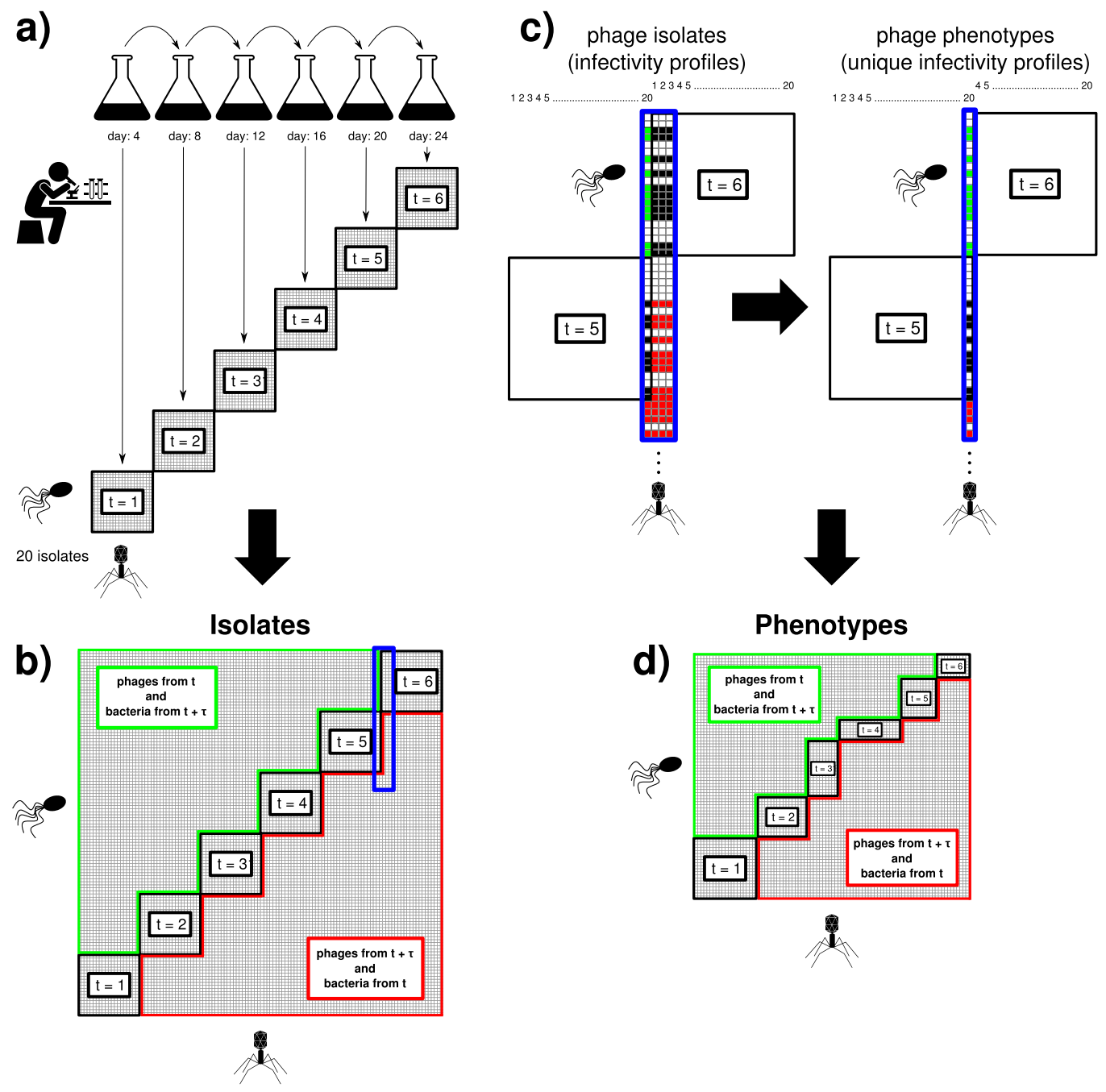

Figure 1: Experimental coevolution. 
Figure 1: Experimental coevolution. a) Coevolving bacteria and phages: 20 bacteria and 20 phages were isolated every 4 days from 12 populations that were coevolving for 24 days in 2 different nutrient environments (6 with high and 6 with low resource availability). b) Cross-infection matrices obtained at the end of the experiment: 6 20x20 matrices of bacteria and phages isolated from the same point in time are represented along the diagonal (black). Below the diagonal (red), pairwise cross-infections between bacteria isolated at earlier points in time than phages are shown. Above the diagonal (green), pairwise cross-infections between bacteria isolated at later points in time than phages are represented. In blue, a selection of 4 phage infectivity profiles are highlighted. c) Infectivity profiles (columns) of the $20+20$ phages isolated at time $t=5$ and $t=6$ and obtained after crossing them with the 120 bacteria isolates (rows) are represented (only the crossinfection patterns of 4 phage and 45 bacteria isolates are shown for illustrative purposes). The infectivity profile of phage $\# 20$ isolated at time $\mathrm{t}=5$ and the infectivity profiles of phages $\# 1, \# 2$, and $\# 3$ isolated at time $\mathrm{t}=6$ are all the same. When this happened, we only kept in the global networks the infectivity profile of the phage isolated at the earliest point in time, and discarded the rest. Changes in the size of the matrices along the diagonal can happen as a result of this process. We applied the same procedure to obtain unique bacteria resistance profiles (rows). d) The resulting cross-infection matrix of unique infectivity/resistance profiles (i.e., phage/bacteria phenotypes) is shown. We use these cross-infection matrices in our analysis (i.e., we worked at the phenotype level). 


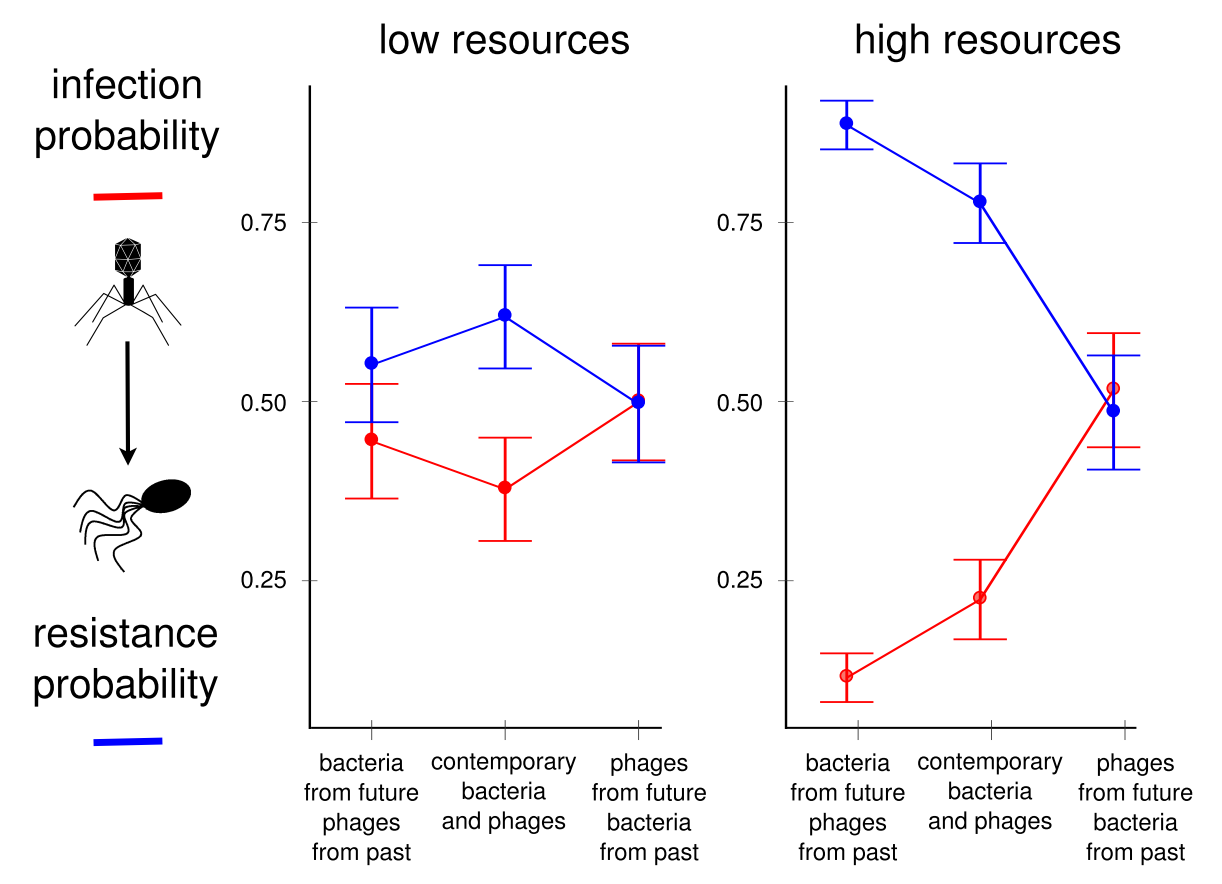

Figure 2: Coevolutionary dynamics. Phage infectivity (red) and bacterial resistance (i.e., 1 - infectivity; blue) at the phenotype level was computed for contemporary bacteria and phages (i.e., both were isolated for the first time at the same point in time), and when bacteria (phages) were facing phages (bacteria) either from the past or the future through time-shifts experiments. Mean and confidence intervals at $95 \%$ of infection and resistance probabilities are shown for low and high resources (for all replicates). Under low resources, bacteria were more resistant to contemporary than to non-contemporary phages and phages were less virulent to contemporary than to non-contemporary bacteria. This result is consistent with fluctuating dynamics when bacteria adapt more rapidly than do phages. In contrast, at high resources bacteria (phages) were more resistant (virulent) to phages (bacteria) from the past than to contemporary phages (bacteria), and to contemporary phages (bacteria) than to phages (bacteria) from the future. This result indicates an ever-increasing reciprocal investment in defense and counterdefense over time (i.e., arms race dynamics). 

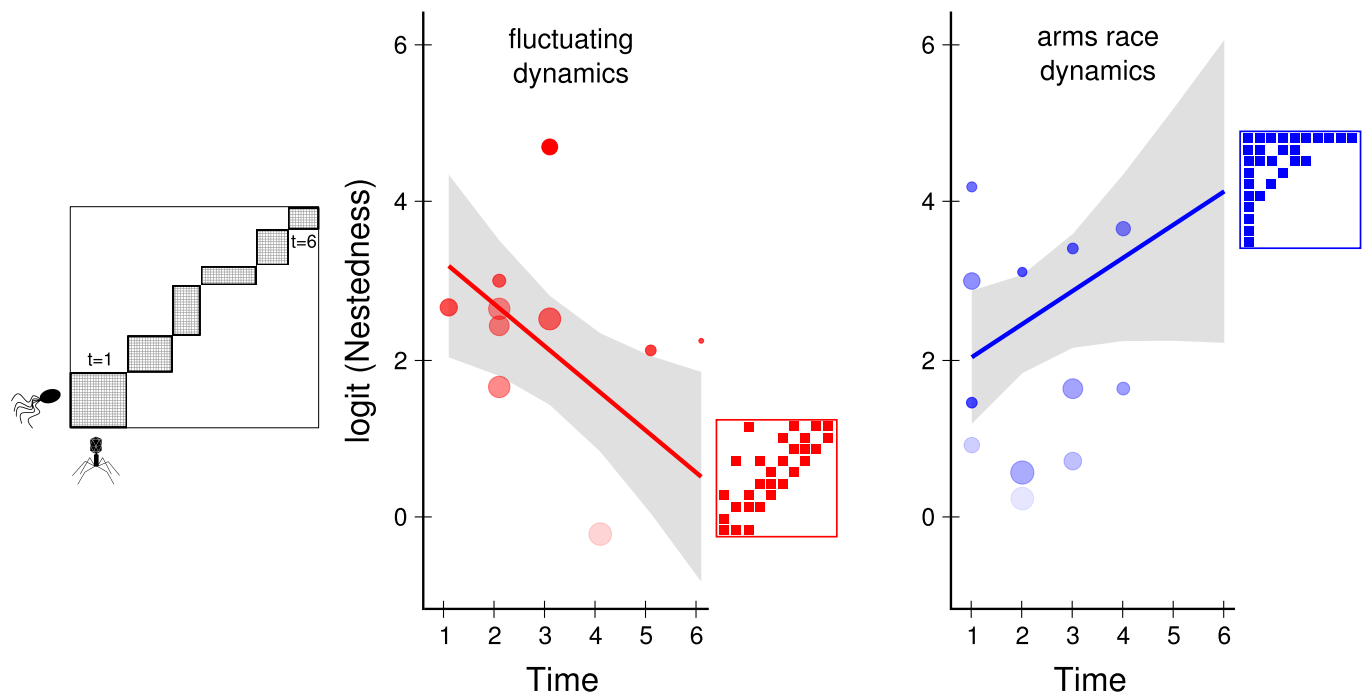

Figure 3: Nestedness of contemporary networks over time. We computed the nested pattern of infection among bacteria and phages that were isolated in the lab at the same point in time (cartoon on the left). Each circle corresponds to a contemporary network, and its diameter is proportional to network size (measured as the number of phages multiplied by the number of bacteria). The darker the color of the circle, the higher the average infectivity (i.e., connectance). Regression lines represent how coevolutionary dynamics affect nestedness over time at the average level of connectance and network size (shaded areas indicate the confidence intervals at 95\%). Cartoons at the right of the regression lines illustrate the infection patterns corresponding to the nestedness values predicted at the last point in time for hypothetical contemporary networks with the same level of connectance $(C=0.3)$. Nestedness decreased over time under fluctuating dynamics (red; left) and increased over time under arms race dynamics (blue; right). 

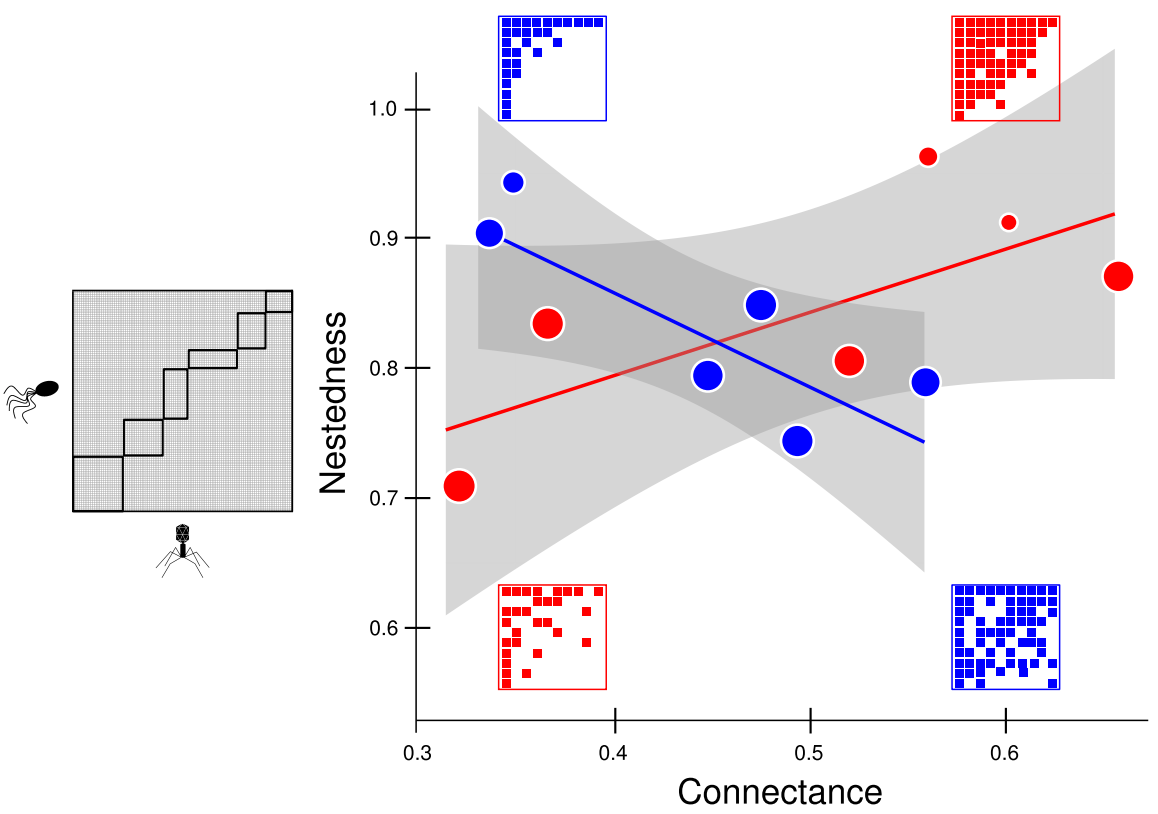

Figure 4: Nestedness of the global network. We computed the nested pattern of infections among all bacteria and phages resulting from the entire coevolutionary experiment (global network with contemporary and non-contemporary bacteria and phages; cartoon on the left). Each circle corresponds to a replicate under fluctuating dynamics (red) and arms race dynamics (blue). The diameter of each circle is proportional to network size (measured as the number of phages multiplied by the number of bacteria). Lines represent the regression lines of the best fit of a generalized linear model (shaded gray areas indicate the confidence intervals at $95 \%$ ). The average infectivity of the network (i.e., connectance) was different across replicates regardless of the mode of coevolution. Cartoons in the corners of the figure illustrate the infection patterns corresponding to the nestedness value for hypothetical networks with high and low connectances $(C=0.6$ and $C=0.3$, respectively). Nestedness increased with connectance under fluctuating dynamics, but decreased under arms race dynamics. 